\title{
Reducing the impact of insulin sensitivity variability on glycaemic outcomes using separate stochastic models within the STAR glycaemic protocol
}

Felicity Thomas ${ }^{1}$, Christopher G Pretty ${ }^{1}$, Liam Fisk¹, Geoffrey M Shaw², J Geoffrey Chase ${ }^{1}$ and Thomas Desaive ${ }^{3^{*}}$

\footnotetext{
* Correspondence: tdesaive@ulg.ac.be

${ }^{3}$ Thermodynamics of Irreversible

Processes, Institute of Physics, Allée du 6 Août, 17 (Bât B5), B4000 Liège, Belgium

Full list of author information is available at the end of the article
}

\begin{abstract}
Background: The metabolism of critically ill patients evolves dynamically over time. Post critical insult, levels of counter-regulatory hormones are significantly elevated, but decrease rapidly over the first 12-48 hours in the intensive care unit (ICU). These hormones have a direct physiological impact on insulin sensitivity (SI). Understanding the variability of SI is important for safely managing glycaemic levels and understanding the evolution of patient condition. The objective of this study is to assess the evolution of SI over the first two days of ICU stay, and using this data, propose a separate stochastic model to reduce the impact of SI variability during glycaemic control using the STAR glycaemic control protocol.
\end{abstract}

Methods: The value of SI was identified hourly for each patient using a validated physiological model. Variability of SI was then calculated as the hour-to-hour percentage change in SI. SI was examined using 6 hour blocks of SI to display trends while mitigating the effects of noise. To reduce the impact of SI variability on achieving glycaemic control a new stochastic model for the most variable period, 0-18 hours, was generated. Virtual simulations were conducted using an existing glycaemic control protocol (STAR) to investigate the clinical impact of using this separate stochastic model during this period of increased metabolic variability.

Results: For the first 18 hours, over $80 \%$ of all SI values were less than $0.5 \times 10^{-3} \mathrm{~L} / \mathrm{mU}$. min, compared to $65 \%$ for $>18$ hours. Using the new stochastic model for the first 18 hours of ICU stay reduced the number of hypoglycaemic measurements during virtual trials. For time spent below 4.4, 4.0, and $3.0 \mathrm{mmol} / \mathrm{L}$ absolute reductions of $1.1 \%, 0.8 \%$ and $0.1 \%$ were achieved, respectively. No severe hypoglycaemic events (BG $<2.2 \mathrm{mmol} / \mathrm{L}$ ) occurred for either case.

Conclusions: SI levels increase significantly, while variability decreases during the first 18 hours of a patients stay in ICU. Virtual trials, using a separate stochastic model for this period, demonstrated a reduction in variability and hypoglycaemia during the first 18 hours without adversely affecting the overall level of control. Thus, use of multiple models can reduce the impact of SI variability during model-based glycaemic control.

Keywords: Insulin sensitivity, Intensive care, Glycaemia, Model-based control 


\section{Introduction}

The metabolism of critically ill patients evolves dynamically over time [1-3]. Post critical insult, plasma concentrations of counter-regulatory hormones such as cortisol, glucagon, growth hormone, and the catecholamines are significantly elevated, but decrease rapidly over the first $12-48$ hours in the intensive care unit (ICU) [2-5]. These hormones have a direct physiological impact on insulin sensitivity (SI) [3]. Therefore, SI is likely to be lowest during the first 12-48 hours in the ICU and increase over time [6-8]. Understanding the variability of SI, over hours and days, is important for safely and effectively managing glycaemic levels, as well as for understanding the evolution of patient condition.

Bagshaw et al. [9] reported associations between mortality, hypoglycaemia, and variability during the first 24 hours of ICU stay. Several other studies [10-13] have also shown that glycaemic variability is independently associated with mortality in critically ill patients. Accurate predictions of SI and its variability would thus enable safer and more effective glycaemic control.

The STAR (Stochastic TARgeted) glycaemic control protocol, has been used in Christchurch Hospital ICU since 2012 [14]. This protocol uses a physiological glucoseinsulin system model coupled with stochastic models of SI variability $[15,16]$ to determine the most appropriate insulin and nutrition treatment combinations. SI is an important consideration in clinical blood glucose (BG) control as it captures the overall glycaemic response of a body to exogenous insulin and nutrition inputs. This measure of glycaemic response to exogenous inputs is particularly important for STAR which aims to minimise the risk of hypoglycaemia by directly accounting for likely variability of SI.

We propose that by using multiple stochastic models, specific to patient condition, performance of the STAR protocol could be enhanced by directly accounting for periods of increased SI variability. With only a small alteration to the controller, use of different stochastic models based on time or diagnosis could allow the existing STAR protocol to achieve more accurate control and/or reduce hypoglycaemia. The objective of this study is to assess the evolution of SI in both magnitude and variability over the first few days of ICU stay, and thus propose and test appropriate additional stochastic models to reduce the impact of SI variability on achieving glycaemic control within the STAR protocol.

\section{Subjects and methods}

\section{Patients}

This study used data from 371 patients admitted to the Christchurch Hospital ICU between 2005 and 2007 and treated with the SPRINT (Specialised Relative Insulin Nutrition Tables) glycaemic control protocol [17]. The variability study and the virtual trials were conducted on two different sub sets of patients from this cohort. Table 1 presents a summary of the cohort demographics. The Upper South Regional Ethics Committee, New Zealand granted approval for the audit, analysis and publication of this data.

\section{Analysis of SI variability \\ Patients}

The analysis of SI variability was performed on a sub-cohort of 164 patients from the SPRINT study [17]. These particular patients were included because SPRINT was 
Table 1 Cohort and Sub-cohort summary statistics

\begin{tabular}{lccc}
\hline & $\begin{array}{c}\text { All } \\
\text { patients }\end{array}$ & $\begin{array}{c}\text { Patients that started SPRINT within } \\
\mathbf{1 8} \text { hrs of ICU admission (used to } \\
\text { generate Stochastic Model) }\end{array}$ & $\begin{array}{c}\text { Patients that started SPRINT with } \\
\text { in 12 hrs of ICU admission and } \\
\text { continued for at least 24 hrs (used } \\
\text { to test separate stochastic model) }\end{array}$ \\
\hline $\begin{array}{l}\text { Number } \\
\text { Age (years) }\end{array}$ & $65[49-74]$ & 287 & 164 \\
$\begin{array}{l}\text { Gender (M/F) } \\
\text { APACHE II score }\end{array}$ & $236 / 135$ & $65[55-74]$ & $65[56-74]$ \\
$\begin{array}{l}\text { APACHE II } \\
\text { ROD (\%) }\end{array}$ & $26[15-24]$ & $181 / 106$ & $102 / 62$ \\
$\begin{array}{l}\text { Operative/ } \\
\text { Non- operative }\end{array}$ & $170 / 201$ & $18[14-24]$ & $19[16-25]$ \\
$\begin{array}{l}\text { Diabetic status } \\
\text { (T1DM/T2DM) }\end{array}$ & $14 / 49$ & $26[13-44]$ & $32[17-52]$ \\
$\begin{array}{l}\text { Hospital Mortality } \\
\text { ICU length of } \\
\text { stay (hrs) }\end{array}$ & $16 \%$ & $143 / 144$ & $66 / 98$ \\
\hline
\end{tabular}

commenced within 12 hours of ICU admission and continued for at least 24 hours, ensuring that enough data was available to accurately assess the change in SI during the first 24 hours of glycaemic control.

\section{Methods}

SI was identified hourly for each patient using the validated Intensive Control InsulinNutrition-Glucose (ICING) model [18]. This is the same model used in the model-based STAR protocol [14]. Variability of SI was calculated as the hour-to-hour percentage change in SI $(\Delta \% \mathrm{SI})$ :

$$
\Delta \% \mathrm{SI}=100 \frac{\left(S I_{k+1}-S I_{k}\right)}{S I_{k}}
$$

The use of percentage change, rather than absolute change, normalises the metric so patients with differing SI levels can be compared fairly.

Bagshaw et al. [9] reported an association between both hypoglycaemia and variability with mortality during the first 24 hours of ICU stay. Thus, the acute evolution of SI over the first day using 6-hour blocks was analysed. For the cohort analysis, SI and $\Delta \%$ SI data from all patients was grouped into each appropriate time-block. Median values for each time-block were calculated for comparison to the previous block, thus capturing overall cohort changes over time in level and hour-to-hour variability.

For the per-patient analysis, the median value of SI and the interquartile range (IQR) of $\Delta \% \mathrm{SI}$ were calculated for each patient, for each time-block. The IQR captures the width of degree of variability of a given patient within each 6-hour block. Thus, a reduction in the IQR of $\Delta \%$ SI over time would indicate a reduction in hour-to-hour variability for a given patient.

Analyses were based on the time spent on the SPRINT protocol, rather than time spent in the ICU, to ensure sufficient insulin and nutrition data to accurately identify an hourly value of SI [19]. Therefore, day 1 comprises the first 24 hours of SPRINT. However, patients were only included in this sub-cohort if they commenced SPRINT 
within 12 hours of ICU admission. Thus, this data is representative of the first day of ICU stay. The median delay between admission and commencement of SPRINT for this cohort was 1.9 hours and $81 \%$ of the cohort $(\mathrm{N}=134)$ had commenced SPRINT within 6 hours of admission, which is well within the reported 24 hour increased variability window [9].

SI levels and variability were compared using cumulative distribution functions (CDFs) and non-parametric statistics. All distributed data was compared using the Wilcoxon rank-sum test (Mann-Whitney U-test), except for SI variability results. SI variability was compared using the Kolmogorov-Smirnov test as it has greater power to detect differences in the shape of distributions when median values are similar. P-values $<0.05$ were considered statistically significant.

\section{Virtual trial simulation and star protocol Patients}

For this virtual trial, and based on the results of the variability analysis, a new stochastic model based only on data collected during the first 18 hours of patient stay in the ICU was used. Thus, data from 287 patients who commenced SPRINT within 18 hour of ICU admission was used. Specifically, if a patient commenced SPRINT 13 hours after ICU admission, only data from the first 5 hours of glycaemic control would contribute to the 0-18 hours stochastic model, and the remainder to the 18+ hours model. These 287 patients where then used in simulation to investigate the potential benefits of using two stochastic models.

\section{Methods}

The virtual trial simulation method used in this study is described in detail by Chase et al. [20]. This method involves using the SI profile of patients, identified from actual clinical data, as the underlying basis for virtual patients. During a virtual trial, a control algorithm is used to select an insulin and nutrition intervention, and the known SI profile is used to simulate the resulting BG profile and take a virtual BG measurement at the next intervention time. Prior work has validated this methodology [20], and this information can be used with the STAR controller to assess the benefits of using separate stochastic models. Cohort statistics such as percentage time in a target band and BG distributions achieved highlight the potential effects of changes to the control algorithm.

The STAR protocol recommends insulin and nutrition interventions based on the predicted BG response over 1-3 hour intervals using a forecasted SI from the stochastic model, as shown in Figure 1 [14]. The STAR protocol targets a BG range by maximising the likelihood of achieving that range, given constraints such as acceptable risk of hypoglycaemia and limitations on insulin and nutrition delivery. If BG is stable, the STAR protocol allows 2 and 3-hour BG measurement options. The STAR protocol is described in detail by Fisk et al. [14,21]. For this analysis, the in-silico STAR controller always selects the longest available measurement interval, to obtain the best balance between the level of control and expected nurse workload.

Currently, the STAR protocol uses a single stochastic model for all patients, for the entire duration of control (the general model) [21]. Hence, this method has no temporal variability. Based on the results of the variability analysis presented in this study, a 


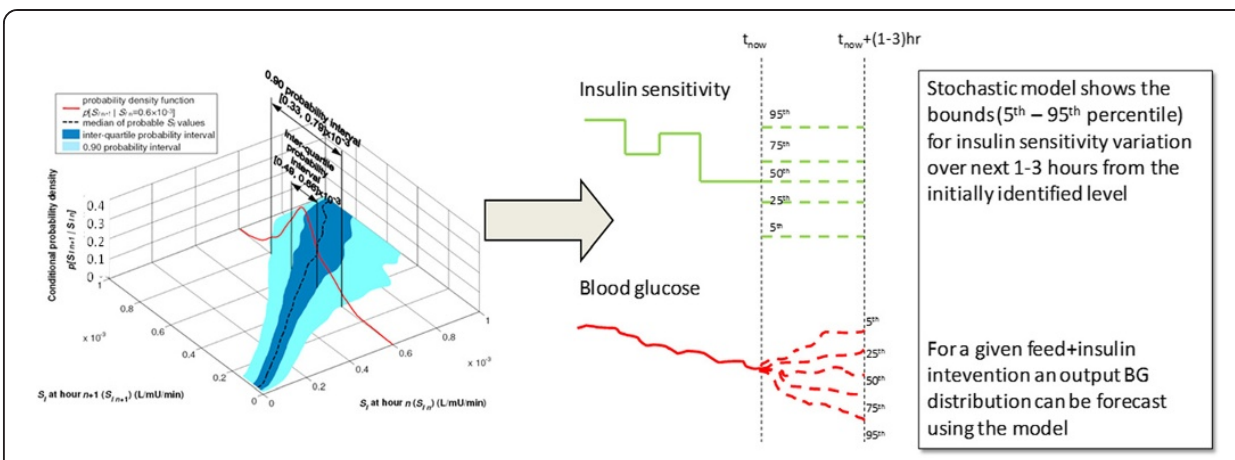

Figure 1 Schematic of the stochastic model showing the percentiles (5th - 95th) for insulin sensitivity variation for the forthcoming $1-3$ hours $(n+1)$. For a feed and insulin intervention an output BG distribution can be forecast using the physiological glucose - insulin model.

specific model was generated from data collected during the first 18 hours of patient stay in the ICU, and used only during this period. An 18+ hour model covers all subsequent time. During virtual trial simulation, if a virtual-patient commences control 13 hours after admission, only the first 5 hours would be controlled using the $0-18$ hour stochastic model.

This study compares outcome glycaemia from virtual trials during the first 18 hours of ICU stay. The 18+ hour stochastic model is sufficiently similar to the general model that comparing results from virtual trials using these two models would show no appreciable difference. Further, by analysing and presenting only the data from the first 18 hours of patient stay (4398 hours for the cohort), changes to the outcome glycaemia are clear, as they are not overwhelmed by the much larger quantity of data post-18 hours (27792 hours for the cohort).

\section{Results}

\section{Insulin sensitivity level and variability}

In addition to the actual insulin sensitivity, the SI parameter also captures any sensor noise and un- or under-modelled processes, such as variability in hepatic glucose output. Therefore, Insulin Sensitivity was examined using 6-hour blocks of SI to display trends while reducing any noise effects through averaging. SI increased over each 6-hour block during the first 24 hours of data, while SI variability decreased over this period. The top half of Table 2 displays the percentage increase in median SI for both the overall cohort and per-patient analyses. It is clear from this table that the first 18 hours result in the largest increase in SI level.

The bottom half of Table 2 shows the reduction in SI variability over time. These values represent the percentage change to the IQR-width of $\Delta \% \mathrm{SI}$ and the shift at the median of per-patient IQR-widths (see Figure 2), for the cohort and per-patient analyses, respectively. Again, the largest change is seen over the first 18 hours. Past 18 hours, both variability and magnitude of SI are similar to that seen on days 2, 3 and 4 (results not presented here) [8].

Figure 2 summarises the difference in SI and variability observed during the first 18 hours of patient data compared to the rest of their stay. For data collected during the first 18 hours of stay, more than $80 \%$ of all SI values were less than $0.5 \times 10^{-3} \mathrm{~L} / \mathrm{mU} \cdot \mathrm{min}$, 
Table 2 The top half of the table displays the increasing cohort and per patient median insulin sensitivity over $6 \mathrm{hr}$ blocks and the second half of the table shows the reductions in the IQR and median per-patient hour to hour percentage insulin sensitivity over time

\begin{tabular}{|c|c|c|c|c|}
\hline \multirow[t]{2}{*}{ SI level analysis } & \multicolumn{2}{|c|}{ Cohort analysis } & \multicolumn{2}{|c|}{ Per-patient analysis } \\
\hline & $\%$ increase at median & p-value & $\%$ increase at median & p-value \\
\hline $0-6$ vs $6-12 \mathrm{hrs}$ & 42 & $<0.0001$ & 40 & 0.0007 \\
\hline $6-12$ vs $12-18$ hrs & 28 & $<0.0001$ & 26 & 0.0123 \\
\hline $12-18$ vs $18-24$ hrs & 1 & 0.0335 & 3 & 0.4822 \\
\hline $18-24$ vs $24-48$ hrs & 9 & 0.0428 & 7 & 0.2873 \\
\hline Variability analysis & $\%$ Change of IQR & p-value & $\%$ Change at median & p-value \\
\hline $0-6$ vs $6-12 \mathrm{hrs}$ & -36 & 0.0092 & -39 & $<0.0001$ \\
\hline $6-12$ vs $12-18 \mathrm{hrs}$ & -24 & 0.0806 & -29 & 0.0794 \\
\hline $12-18$ vs $18-24 \mathrm{hrs}$ & 1 & 0.0806 & -9 & 0.1029 \\
\hline $18-24$ vs $24-48$ hrs & -19 & 0.0998 & -18 & 0.0467 \\
\hline
\end{tabular}

P-values calculated using Kolmogorov-Smirnov test for cohort comparisons and Wilcoxon rank-sum for per-patient comparisons.

compared to $65 \%$ for $>18$ hours. Both per patient panels (B and D) show the same trends as the whole-cohort results, with the $0-18$ hour model exhibiting a higher SI level $\left(80 \%<0.4 \times 10^{-3} \mathrm{~L} / \mathrm{mU} \cdot \mathrm{min}\right)$ and greater variability. As the first 18 hours produced the greatest increase in SI level and reduction in variability, this period was selected as the most likely to see benefit from using a separate stochastic model.

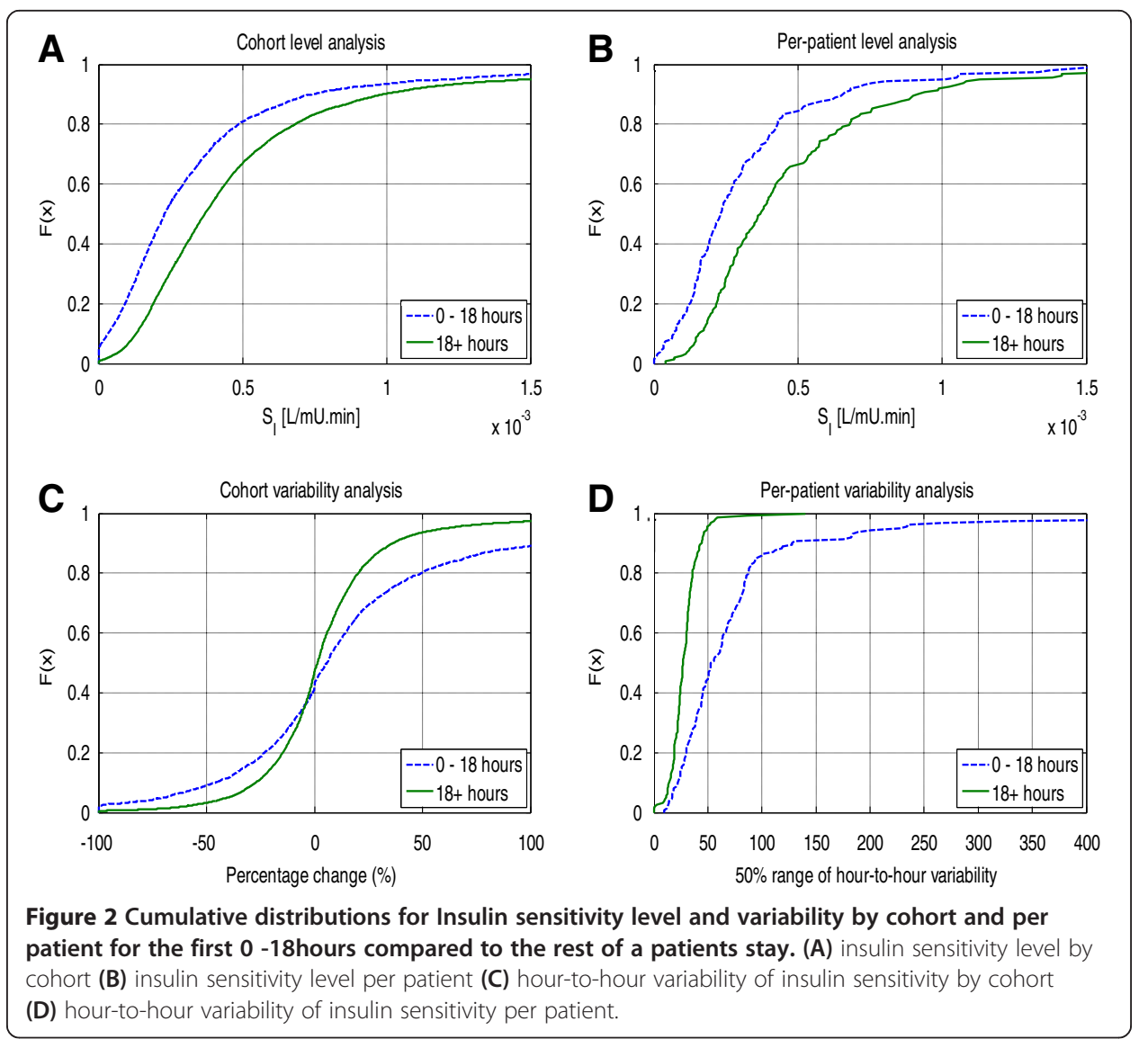




\section{Virtual trials}

Figure 3 compares the $0-18$ hour stochastic model with the general model used in this analysis. The $\mathrm{y}$-axis shows the distribution of expected SI values for hour $\mathrm{n}+1$ given the $\mathrm{x}$-axis value of SI at hour $\mathrm{n}$. The lines on this plot represent the $5^{\text {th }}, 25^{\text {th }}, 50^{\text {th }}, 75^{\text {th }}$ and $95^{\text {th }}$ percentiles. The $0-18$ hour model has noticeably wider intervals between the $5^{\text {th }}-95^{\text {th }}$ percentiles than the general model. This increased width is expected due to the increased hour-to-hour patient variability during the first 18 hours of patient stay and reflects the results presented in Figure 2A and D. Additionally, data points for the 0-18 hour model are concentrated at lower SI levels, also matching the previously reported results.

Figure 4 and Table 3 summarise the results of the virtual trials comparing use of a specific 0-18 hour stochastic model with the general model. These results show a shift in the BG distribution at low BG levels $(<7 \mathrm{mmol} / \mathrm{L})$ when compared to the general model. This result fits with the wider percentile bands of the 0-18 hour model, and represents a small reduction in overall cohort glycaemic variability. Despite this shift, the median BG for the $0-18$ hour model is only $0.2 \mathrm{mmol} / \mathrm{L}$ higher than the general model and is still well within the target band shown in Figure 4. The interquartile range of the per-patient median glycaemia is reduced using the 0-18 hour model, demonstrating the benefit of using this separate stochastic model during a known period of patient variability.

Importantly, the 0-18 hour stochastic model reduces the number of hypoglycaemic measurements. For time spent below 4.4, 4.0 and $3.0 \mathrm{mmol} / \mathrm{L}$ absolute reductions of $1.1 \%, 0.8 \%$ and $0.1 \%$ were achieved, respectively (relative reductions of $20-33 \%$ ). These values represent clinically significant changes $(\mathrm{p}=0.008, \mathrm{p}=0.0098$ and $\mathrm{p}=0.35$, Two-Tailed Fishers Exact Test, respectively), particularly given the association of hypoglycaemia with negative patient outcome [11]. No severe hypoglycaemic events $(\mathrm{BG}<2.2 \mathrm{mmol} / \mathrm{L})$ occurred for either case.

\section{Discussion}

The use of a time-specific 0-18 hour stochastic model reduces hypoglycaemia and glycaemic variability over the first 18 hours of patient admission. This reduction in

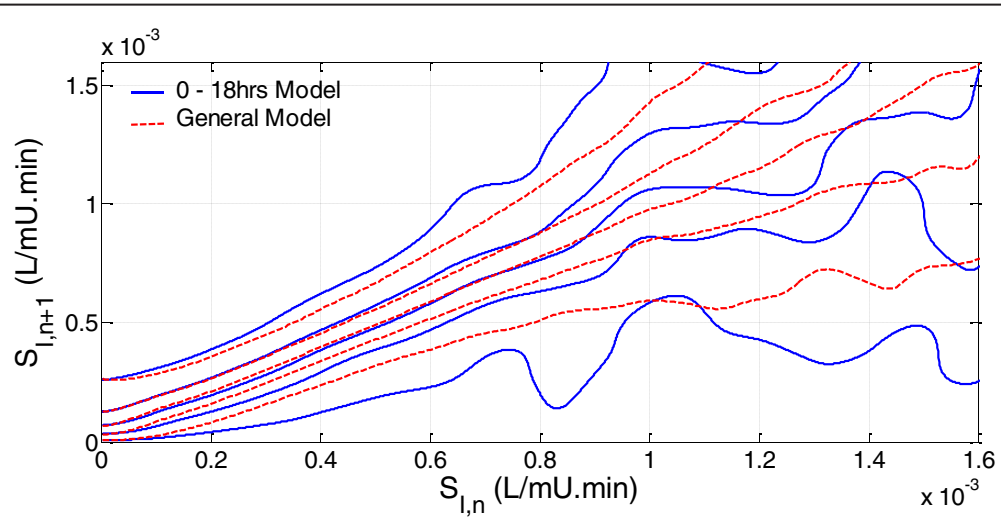

Figure 3 Comparison of $0-18$ hrs and general stochastic models where the lines represent the 5 th, 25th, 50th, 75th and 95th percentiles from the x-axis upward. 


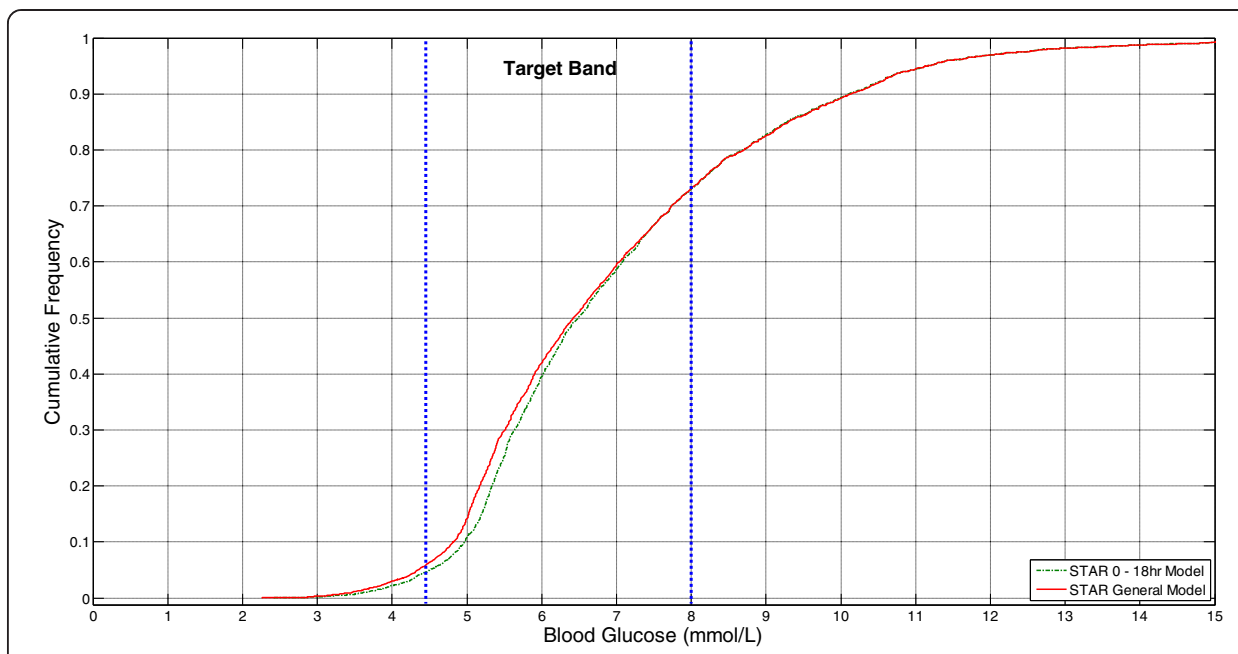

Figure 4 Cumulative distribution of the BG results from the virtual trial simulations performed over the first 18 hours of ICU stay using a specific $0-18 \mathrm{hr}$ stochastic model and the general model, with the STAR protocol.

hypoglycaemia is due to the wider percentile bands of the $0-18$ hour stochastic model, as seen in Figure 3. The STAR protocol constrains the $5^{\text {th }}$ percentile BG prediction to a lower limit, and thus increased width of the percentile bands causes a shift in the BG distribution to higher levels.

Approximately 300-400 patients a year receive glycaemic control from the STAR protocol in Christchurch hospital Intensive Care Unit. These patients are typically treated by the STAR protocol for approximately 5 days, resulting in 24000 BG measurements per year. Hence, a reduction of $\mathrm{BG}<4.4 \mathrm{mmol} / \mathrm{L}$ by $1.1 \%$ could decrease the number of mild-hypoglycaemic events by up to 260 each year.

Additionally, the BG interquartile range was reduced by applying the $0-18$ hour model. This result indicates a small reduction on overall cohort glycaemic variability

Table 3 Virtual trial simulation BG results comparison for data within 18 hours of ICU admission

\begin{tabular}{lcc}
\hline Whole cohort control statistics & STAR general model & STAR $\mathbf{2} \mathbf{0}$-18 hrs model \\
\hline Num Patients & 287 & 287 \\
Total hours & 5591 hours & 5292 hours \\
Num BG measurements & 4232 & 4096 \\
Target BG band (mmol/L) & $4.4-8.0$ & $4.4-8.0$ \\
BG median $[I Q R](\mathrm{mmol} / \mathrm{L})$ & $6.3[5.3-8.1]$ & $6.5[5.5-8.1]$ \\
$\%$ BG within $4.0-6.1 \mathrm{mmol} / \mathrm{L}$ & 45.9 & 42.9 \\
$\%$ BG within $4.4-7.0 \mathrm{mmol} / \mathrm{L}$ & 55.4 & 54.7 \\
$\%$ BG within $4.4-8.0 \mathrm{mmol} / \mathrm{L}$ & 68.7 & 69.0 \\
$\%$ BG within $8.0-10 \mathrm{mmol} / \mathrm{L}$ & 15.7 & 16.1 \\
$\%$ BG $>10 \mathrm{mmol} / \mathrm{L}$ & 10.2 & 10.5 \\
$\%$ BG $<4.4 \mathrm{mmol} / \mathrm{L}$ & 5.5 & 4.4 \\
$\%$ BG $<4.0 \mathrm{mmol} / \mathrm{L}$ & 3.0 & 2.2 \\
$\%$ BG $<3$ mmol/L & 0.3 & 0.2 \\
Num patients $<2.2 \mathrm{mmol} / \mathrm{L}$ & 0 & 0 \\
\hline
\end{tabular}


and may be due to less 'over control' by STAR for variable patients. Hence, these virtual trials indicate that the impact of SI variability can be reduced by using more than one stochastic model. This reduced variability is potentially beneficial as glycaemic variability has been shown to be independently associated with mortality in critically ill patients [10-13]. Therefore, not only does the additional stochastic model improve control performance by reducing the occurrence of hypoglycaemia, it also has the potential to improve patient outcomes by reducing variability.

It has been proposed that a number of factors, including cardiovascular surgery and glucocorticoid therapy, are associated with increased SI variability. Thus it would be ideal to be able to create separate stochastic models for each of these different cases, and potentially separate models for different time frames with in the first 24 hours of ICU stay. However, currently we do not have enough available data to create specific stochastic models for all proposed cases, while ensuring the data used in each is independent of the others. Nevertheless, this study has validated the concept of using additional stochastic models to achieve more accurate glycaemic control with reduced hypoglycaemia.

\section{Conclusions}

Insulin sensitivity levels increase significantly, while variability decreases over the first few days of patient stay in the ICU. This study determined that the largest changes in SI level and variability occur during the first 18 post ICU admission, and thus, a separate stochastic model of SI behaviour for this period is warranted for use with model-based controllers to better manage this evolution.

Virtual trials using a separate stochastic model for the first 18 hours of stay demonstrated a reduction in both glycaemic variability and hypoglycaemia during this period without adversely affecting the overall level of control. Thus, use of multiple models can reduce the impact of SI variability during model-based glycaemic control. As more data becomes available this same method could be used to analyse and generate stochastic models specific to various diagnostic categories, drug therapies or other situations that are thought to increase metabolic variability.

\section{Key message}

The impact of Insulin Sensitivity variability on glycaemic outcomes can be reduced by using separate stochastic models.

\section{Abbreviations}

ICU: Intensive care unit; SI: Insulin sensitivity; BG: Blood glucose; ICING: Intensive control insulin-nutrition-glucose; STAR: Stochastic TARgeted; SPRINT: Specialised relative insulin nutrition tables; IQR: Interquartile range.

Competing interests

The authors declare that they have no competing interests.

\section{Authors' contributions}

FT carried out the analysis of SI variability and the stochastic model generation and simulation. CP carried out an initial study in to SI variability and over saw the design and coordination of this follow up study. LF assisted with the generation of a new stochastic model and simulation of this model. GS provided clinical insight and supervised the acquisition of all clinical data used. JGC conceived of the study, and participated in its design. TD participated in the design and coordination of this study. All authors read and approved the final manuscript. 


\section{Author details}

'Department of Mechanical Engineering, University of Canterbury, Private Bag 8140, Christchurch, New Zealand.

${ }^{2}$ Department of Intensive Care, Christchurch Hospital, Private Bag 4710, Christchurch, New Zealand. ${ }^{3}$ Thermodynamics of Irreversible Processes, Institute of Physics, Allée du 6 Août, 17 (Bât B5), B4000 Liège, Belgium.

Received: 29 January 2014 Accepted: 1 April 2014

Published: 16 April 2014

\section{References}

1. Cuthbertson DP: Post-shock metabolic response. Lancet 1942, 1:433-437.

2. Frayn KN: Hormonal-control of metabolism in trauma and sepsis. Clin Endocrinol 1986, 24(5):577-599.

3. Weissman C: The metabolic response to stress: an overview and update. Anesthesiology 1990, 73(2):308-327.

4. Chernow B, Zaloga GP: Pharmacologic management of the critically ill patient in the perioperative periodemphasis on the sepsis syndrome. Med Clin North Am 1987, 71(3):541-549.

5. Jaattela A, Alho A, Avikainen V, Karaharju E, Kataja J, Lahdensuu M, Lepisto P, Rokkanen P, Tervo T: Plasma catecholamines in severely injured patients: a prospective study on 45 patients with multiple injuries. $\mathrm{Br} J$ Surg 1975, 62(3):177-181.

6. Ferenci T, Benyo B, Kovacs L, Fisk L, Shaw GM, Chase JG: Daily evolution of insulin sensitivity variability with respect to diagnosis in the critically ill. PLoS One 2013, 8(2):e57119.

7. Langouche L, Vander Perre S, Wouters PJ, D'Hoore A, Hansen TK, Van den Berghe G: Effect of intensive insulin therapy on insulin sensitivity in the critically ill. J Clin Endocrinol Metab 2007, 92(10):3890-3897.

8. Pretty CG, Le Compte AJ, Chase JG, Shaw GM, Preiser JC, Penning S, Desaive T: Variability of insulin sensitivity during the first 4 days of critical illness: implications for tight glycemic control. Ann Intensive Care 2012, 2(1):17

9. Bagshaw SM, Egi M, George C, Bellomo R: Early blood glucose control and mortality in critically ill patients in Australia. Crit Care Med 2009, 37(2):463-470.

10. Egi M, Bellomo R, Stachowski E, French CJ, Hart G: Variability of blood glucose concentration and short-term mortality in critically ill patients. Anesthesiology 2006, 105(2):244-252.

11. Egi M, Bellomo R, Stachowski E, French CJ, Hart GK, Taori G, Hegarty C, Bailey M: Hypoglycemia and outcome in critically ill patients. Mayo Clin Proc 2010, 85(3):217-224.

12. Hermanides J, Vriesendorp TM, Bosman RJ, Zandstra DF, Hoekstra JB, Devries JH: Glucose variability is associated with intensive care unit mortality. Crit Care Med 2010, 38(3):838-842.

13. Krinsley JS: Glycemic variability: a strong independent predictor of mortality in critically ill patients. Crit Care Med 2008, 36(11):3008-3013.

14. Fisk LM, Le Compte AJ, Shaw GM, Penning S, Desaive T, Chase JG: STAR development and protocol comparison. IEEE Trans Biomed Eng 2012, 59(12):3357-3364.

15. Lin J, Lee D, Chase JG, Shaw GM, Le Compte A, Lotz T, Wong J, Lonergan T, Hann CE: Stochastic modelling of insulin sensitivity and adaptive glycemic control for critical care. Comput Methods Prog Biomed 2008, 89(2):141-152.

16. Lin J, Lee D, Chase J, Hann C, Lotz T, Wong X: Stochastic modelling of insulin sensitivity variability in critical care. Biomed Signal Process Control 2006, 1:229-242.

17. Chase JG, Shaw G, Le Compte A, Lonergan T, Willacy M, Wong X-W, Lin J, Lotz T, Lee D, Hann C: Implementation and evaluation of the SPRINT protocol for tight glycaemic control in critically ill patients: a clinical practice change. Crit Care 2008, 12(2):R49.

18. Lin J, Razak NN, Pretty CG, Le Compte A, Docherty P, Parente JD, Shaw GM, Hann CE, Chase JG: A physiological Intensive Control Insulin-Nutrition-Glucose (ICING) model validated in critically ill patients. Comput Methods Prog Biomed 2011, 102(2):192-205.

19. Hann CE, Chase JG, Lin J, Lotz T, Doran CV, Shaw GM: Integral-based parameter identification for long-term dynamic verification of a glucose-insulin system model. Comput Methods Programs Biomed 2005, 77(3):259-270.

20. Chase JG, Suhaimi F, Penning S, Preiser JC, Le Compte AJ, Lin J, Pretty CG, Shaw GM, Moorhead KT, Desaive T: Validation of a model-based virtual trials method for tight glycemic control in intensive care. Biomed Eng Online 2010, 9:84

21. Evans A, Shaw GM, Le Compte A, Tan CS, Ward L, Steel J, Pretty CG, Pfeifer L, Penning S, Suhaimi F, Signal M, Desaive T, Chase JG: Pilot proof of concept clinical trials of Stochastic Targeted (STAR) glycemic control. Ann Intensive Care 2011, 1:38

doi:10.1186/1475-925X-13-43

Cite this article as: Thomas et al:: Reducing the impact of insulin sensitivity variability on glycaemic outcomes using separate stochastic models within the STAR glycaemic protocol. BioMedical Engineering OnLine 2014 13:43. 